\title{
Periodontal disease and its prevention, by traditional and new avenues (Review)
}

\author{
JONATHAN MANN ${ }^{1}$, YARON BERNSTEIN ${ }^{2}$ and MOTI FINDLER ${ }^{2}$ \\ ${ }^{1}$ Department of Community Dentistry, Faculty of Dental Medicine, Hebrew University-Hadassah School of \\ Dental Medicine, 91120 Jerusalem; ${ }^{2}$ MACCABIDENT-Dental Health Service, 6801298 Tel Aviv, Israel
}

Received August 21, 2019; Accepted November 18, 2019

DOI: 10.3892/etm.2019.8381

\begin{abstract}
One of the most prevalent diseases affecting humans are those which affect the oral cavity. Several diseases can affect the mouth, such as oral cancer, dental caries, Lichen Planus and, of course, periodontal disease with its variants. This review discusses gingivitis, periodontitis and peri-implantitis with a brief focus on the different primary prevention aids, such as mechanical, chemical and the new generation of potential products to be used in the future dental market, products that are prepared from natural sources, the general trend worldwide. In this review, not all new inventions will be mentioned, yet, the role of aged garlic extract (AGE) will be described in addition to a new toothpaste which originates from the Dead Sea and the Purecare dental which produces the Ozone (H3) for the prevention of periodontal disease.
\end{abstract}

\section{Contents}

1. Classification of periodontal diseases

2. Primary prevention

3. New inventions

4. Summary

\section{Classification of periodontal diseases}

Periodontal diseases include several forms and symptoms; however, the most common are gingivitis and periodontitis. As dental implants are becoming more common in the routine treatment in dentistry, an additional periodontal disease, peri-implantitis, is increasing in prevalence. It is crucial to

Correspondence to: Professor Jonathan Mann, Department of Community Dentistry, Faculty of Dental Medicine, Hebrew University-Hadassah School of Dental Medicine, Ein Kerem, P.O. Box 12000, 91120 Jerusalem, Israel

E-mail: dman@hadassah.org.il

Key words: periodontal disease, aged garlic extract, primary prevention define all three diseases, clarify the etiology and epidemiology, and discuss prevention measures for these diseases.

According to the US National Institute of Dental and Craniofacial Research, periodontal disease is an infection of the tissue that holds one's teeth in place. It is typically caused by poor oral hygiene that allows the sticky film of bacteria, known as plaque, to build up on the teeth and later develop into calculus (1). According to a study published by the Dental Health Foundation of Ireland, gingivitis is characterized by the redness of the gum margins, swelling and bleeding on brushing. Gingivitis occurs in both chronic and acute forms. Acute gingivitis is associated with specific infections, micro-organisms, or trauma (2), and chronic inflammation of the gum tissue surrounding the teeth is associated with the bacterial biofilm that covers the teeth and gums. Periodontitis affects the bone and supporting tissue, and is characterized by the formation of pockets or 'spaces' between the tooth and gums.

The majority of adults are affected by gingivitis. Gingivitis does not always develop into periodontal disease. It is a silent disease and a patient suffering from the disease might only begin to notice when it is already irreversible. The signs of periodontitis may include bleeding during brushing, mobility while eating, for example, bad mouth odor (halitosis) and other signs at a later stage. In order to diagnose periodontal disease, one must be regularly examined by a dentist or a dental hygienist. In the majority of cases, periodontal disease responds to treatment and its progression can be halted although the destruction is largely irreversible (3).

The severity of the attack varies from one individual to another, depending on the virulence of the bacterial plaque and on the efficiency of the local and systemic immune inflammatory response of the person. No doubt the host response is influenced by both environmental and genetic factors. Systemic diseases are an additional factor in determining the severity of periodontal disease. These may include diabetes, leukemia, Down syndrome and others. Other factors associated with periodontal disease are smoking and stress.

A relatively new periodontal disease is peri-implantitis. This disease is a major biological complication of implant therapy, which impairs the long-term success of this procedure (4). The prevalence of peri-implantitis seems to be in the order of $10 \%$ of implants and $20 \%$ of patients during 5-10 years following implant placement (5). 
Periodontitis is widely regarded as the second most common type of dental disease worldwide, following dental decay. It is prevalent at $30-50 \%$ of the population in the United States, but only approxiamtely $10 \%$ of cases are severe forms of the disease (5). Periodontal disease is believed to be associated with various systemic health issues. Increasing evidence points to the fact that diseases, including Alzheimer's, cancer (pancreatic cancer), respiratory diseases, diabetes, hypertension, atherosclerosis and others are associated with gum diseases. It is also believed that the link between heart (myocardial infarction) and gum disease may be due to bacteria. Bacteria in the gums can enter the blood supply and be transferred to distant destinations, including the heart. Other organs which may be affected are the lungs and genital organs, as well as erectile disfunction (6).

\section{Primary prevention}

Periodontal disease is initially a local oral disease with its pathologies including tooth loss, inflammation, difficulty in chewing and bad breath (halitosis). However, it is important to recognize that the disease has a much wider range of impact and potential pathologies which have to draw our attention to its treatment and prevention. Treatment involves good oral hygiene and professional teeth cleaning. In certain cases, it will also include the use of antibiotics and periodontal surgery. No doubt the most important treatment is primary prevention, which is not only most effective, but also the most cost-effective method of coping with the disease and with its complications (7). Primary prevention using toothbrushes or other mechanical aids has been performed for hundreds of years. New devices are introduced by inventors; however, the majority of these, even though some may be effective, do not reach or remain in the dental market for various reason (8).

In addition to the various types of toothbrushes, other mechanical devices, including dental floss, water irrigation (water pick), toothpicks such as the Stim-U-Dent plaque removers, small interproximal brushes, rubber gum stimulator and others have been in the market for years $(9,10)$.

In order to strengthen the effects of the mechanical avenue, an additional chemical, the antiseptic avenue, has been developed, including the chlorhexidine gluconate-based mouthwash, and other mouth rinses, such as Listerine. To the family of chemicals used, hydrogen peroxide should be added, slow release device aids (periochip), antibiotics when needed and the ingredients of toothpastes, such as triclosan, which has recently been replaced by Stannous fluoride $(11,12)$. All these are used in order to eliminate or decrease the bacteria related to plaque buildup and also in order to prevent accumulation of tartar (calculus).

Mouthwashes combine various ingredients for the treatment of a variety of oral conditions; however, these rinses do not replace the need for regular brushing and the use of interproximal aids. They are used as adjuncts to daily home care, by preventing and controlling supragingival plaque, gingivitis and oral malodor (10). The consumer guide to dentistry divides the rinses into three groups (13) as follows: Cosmetic oral rinses may temporarily control or reduce halitosis, rinse oral debris, diminish bacteria and leave one with a refreshing taste. The other group is the therapeutic oral rinses, which are anti plaque and anti-gingivitis or anti-cavity and the third group is the natural/herbal oral rinses (14). A detailed description of all rinses and their active ingredients will necessitate an additional manuscript. Prevention aids, mechanical and chemical, of periodontal diseases, change and improve from day to day.

\section{New inventions}

Investigations are on the way with a number of novel ideas, three of which seem to be very promising. The one is the YAMA+ natural toothpaste based on $70 \%$ Dead Sea water and $30 \%$ essential oils with anti- inflammatory attributes. Salt has a long history of beneficial effects on healing gum disease (15). The new product, is currently undergoing clinical trials.

The second is aged garlic extract (AGE) manufactured by the Wakunaga Company of Japan. AGE is manufactured from garlic which has been used for medical purposes since 3000 B.C. Garlic was used by the Soviets in World War II to prevent infections, and when antibiotics were not available, it was used as a replacement, and was hence termed 'Russian Penicillin', while others refer to it as the 'Miraculous Herb'. AGE is an odorless product resulting from the prolonged extraction of fresh garlic and is highly bioavailable and exhibits biological activity both in animals and humans. Several studies have investigated the effects of AGE on various diseases, as published in the proceedings (please see studies included in this Special Issue of Experimetnal and Therapeutic Medicine); yet, to date, the effects on the oral cavity have not been studied in humans, at least to the best of our knowledge.

A clinical trial which was published in 2018, demonstrated that the daily consumption of AGE, benefited oral health by reducing gingival inflammation and gingival bleeding as compared to a placebo control (16). The results of the study indicated that the AGE group exhibited significantly better results, both when using the Modified Gingival Index (MGI) and the Gingival Bleeding Index (GBI), as compared to the control (placebo) group following the four-month study. An additional study which has just been completed and is not published yet, evaluated the loss of attachment following an 18-month use of AGE (unpublished data). In that study, the level of periodontitis was found to be significantly lower in the AGE group as compared to the placebo group. These two above-mentioned studies indicate that AGE may become a significant player in the world of prevention of gingival and periodontal diseases.

Ozone therapy is a form of alternative medicine that purports to increase the amount of oxygen in the body through the introduction of ozone. Ozonated water is a natural disinfectant, biocompatible with oral tissue. The ozonated water reduces inflammation and kills pathogenic bacteria. The Purecare oral irrigator is a new method through which regular water with a patented capsule and by a simple procedure produce irrigatable Ozone $(17,18)$. This has recently been introduced into the market.

\section{Summary}

This literature review of gingivitis, periodontitis and peri-implantitis is an up-to-date summary evaluating the pathology of the very prevalent diseases and the avenues of 
prevention as they are found today in the market and with some thoughts and ideas for the future.

\section{Acknowledgements}

Not applicable.

\section{Funding}

No funding was received.

\section{Availability of data and materials}

Not applicable.

\section{Authors' contributions}

JM and MF and YB were all involved in the conception and design of this literature review, and they all contributed to the writing and editing of the manuscript, and provided comments during all stages of writing. All authors have read and approved the final manuscript.

\section{Ethics approval and consent to participate}

Not applicable.

\section{Patient consent for publication}

Not applicable.

\section{Competing interests}

The authors declare that they have no competing interests.

\section{References}

1. National Institute of Dental and Craniofacial Research: Periodontal (Gum) Disease. https://www.nidcr.nih.gov/healthinfo/gum-disease/more-info. Accessed July, 2018.

2. Periodontal Disease. Dental Health Foundation, Dublin 2 , 2019. https://www.dentalhealth.ie/dentalhealth/causes/periodontaldisease.html.

3. Irfan UM, Dawson DV and Bissada NF: Epidemiology of periodontal disease: A review and clinical perspectives. J Int Acad Periodontol 3: 14-21, 2001.
4. Wikipedia: Periodontal Disease. https://en.wikipedia. org/wiki/Periodontal_disease.

5. G Caton J, Armitage G, Berglundh T, Chapple ILC, Jepsen S, S Kornman K, L Mealey B, Papapanou PN, Sanz M and S Tonetti M: A new classification scheme for periodontal and peri-implant diseases and conditions - Introduction and key changes from the 1999 classification. J Clin Periodontol 45 (Suppl 20): S1-S8, 2018.

6. Mombelli A, Müller N and Cionca N: The epidemiology of peri-implantitis. Clin Oral Implants Res 23 (Suppl 6): 67-76, 2012.

7. Nazir MA: Prevalence of periodontal disease, its association with systemic diseases and prevention. Int J Health Sci (Qassim) 11: 72-80, 2017.

8. Vos T, Flaxman AD, Naghavi M, Lozano R, Michaud C, Ezzati M Shibuya K, Salomon JA, Abdalla S, Aboyans V, et al: Years lived with disability (YLDs) for 1160 sequelae of 289 diseases and injuries 1990-2010: A systematic analysis for the Global Burden of Disease Study 2010. Lancet 380: 2163-2196, 2012.

9. Newman T: The unexpected dangers of gum disease. Medical News Today, Brighton, East Sussex, 2019. https://www.medicalnewstoday.com/articles/324485.php\#1. Accessed February 22, 2019.

10. Petersen PE and Ogawa H: Strengthening the prevention of periodontal disease: The WHO approach. J Periodontol 76: 2187-2193, 2005.

11. Purtill C: Colgate eliminated triclosan from its toothpaste. Could a ban be on the way? Quartz, New York, NY, 2019. https:// qz.com/1540815/colgate-eliminated-triclosan-from-its-toothpastecould-a-ban-be-on-the-way/. Accessed February 2, 2019.

12. Weatherly LM and Gosse JA: Triclosan exposure, transformation, and human health effects. J Toxicol Environ Health B Crit Rev 20: 447-469, 2017.

13. Cortelli JR and Thenoux RE: The effect of mouth-rinses against oral microorganisms. Braz Oral Res 21 (Spec Iss 1): 23-28, 2007.

14. Department of Scientific Information, ADA Science Institute: Mouthwash (Mouthrinse). ADA, Chicago, IL, 2019. https:// www.ada.org/en/member-center/oral-health-topics/mouthrinse. Accessed August 29, 2019.

15. Condò SG, DeVizio W and Volpe AR: Gingiva, teeth and sea salt. Am J Dent 12: 5-8, 1999.

16. Zini A, Mann J, Mazor S and Vered Y: The Efficacy of Aged Garlic Extract on Gingivitis - A Randomized Clinical Trial. J Clin Dent 29: 52-56, 2018.

17. Wikipedia: Ozone therapy. https://en.wikipedia.org/wiki/Ozone_ therapy.

18. Pure care dental. https://www.purecare.dental.

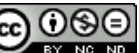

This work is licensed under a Creative Commons Attribution-NonCommercial-NoDerivatives 4.0 International (CC BY-NC-ND 4.0) License. 